\title{
Exploring cancer rehabilitation services in South Wales: qualitative understanding from the perspectives of people affected by cancer and healthcare professionals
}

Judit Csontos, Dr Dominic Roche, Dr Tessa Watts

Purpose:

Internationally, cancer rehabilitation has been found to have positive impact on quality of life (Hunter et al. 2017). Services can consist of physical exercise, psychological support and health education, depending on people's needs. However, 39\% of Welsh Cancer Patient Experience Survey respondents reported they did not always receive practical advice or support to cope with the consequences of cancer (Welsh Government, 2017). The reason for this lack of support and the value of existing services in Wales, UK have not yet been fully investigated. The aim of this study was to explore the value, barriers and facilitators to cancer rehabilitation from the perspectives of people affected by cancer and healthcare professionals.

\section{Methodology:}

A purposive sample of healthcare professionals $(n=20)$ and people affected by cancer $(n=15)$ was recruited from two study sites.

Eligible healthcare professionals had at least one year experience in cancer rehabilitation. People affected by cancer were recruited if they received rehabilitation from one of the study sites. Approval was granted by London South East Research Ethics Committee (17/LO/2123).

Written informed consent was obtained from every participant.

Audio recorded, semi-structured one-onone interviews were transcribed verbatim. Three interviews were dyadic due to people's partner's joining.

Thematic analysis was based on Braun and Clarke (2006).

\section{Good for the body and soul}

Numerous people reported the positive effect of exercise classes on their health. Exercise classes as part of cancer rehabilitation were valuable for restoring physical health. Additionally, cancer rehabilitation had a major role in helping people cope with stress and anxiety related to their cancer diagnosis:

.... do a lot of arm work and lifting weights, which improves my overall wellbeing, because I breathe better, I relax better and I'm learning how to relax again. Oh, dear, I couldn't go to sleep some nights, I just couldn't relax. But now I can. And it's partly the physical exercise I'm doing, coz I'm properly tired and the breathing exercises that I've... I've learned via Tai Chi, so it's... it's coming together.' (Participant_03 affected by breast cancer)

although i... it's hydrotherapy, hm... there is a big social and mental side to it [hydrotherapy]. I would reckon that my problems were somewhere in the region of $60 \%$ mental...' (Participant_10 affected by prostate cancer)

\section{Expert attention}

For people affected by cancer, it was important to have someone who understood their diagnosis and was able to reassure them with expert advice on what they could achieve and how far they could push themselves physically. 'Even when in the pool [hydrotherapy] first I had 'Oh, no' I was a bit shy, I didn't want to. But their reassurance and their coaxing me to do things. I think that's what was the best thing for me. Hm... just putting my trust in them really.' (Participant_28 affected by breast cancer)

'And hm... the girls doing the Tai Chi classes are really, really good, and they're very attentive, because they're watching you all the time and one day I was feeling a bit hm... light headed and hm... I didn't think I was showing it at all. And I said I was fine, and the next thing one of them was next to me saying 'Are you Ok?' And I thought 'How did you know?' (giggling) So watching, you don't realise that they're watching you so much...' (Participant_02 affected by colorectal cancer)

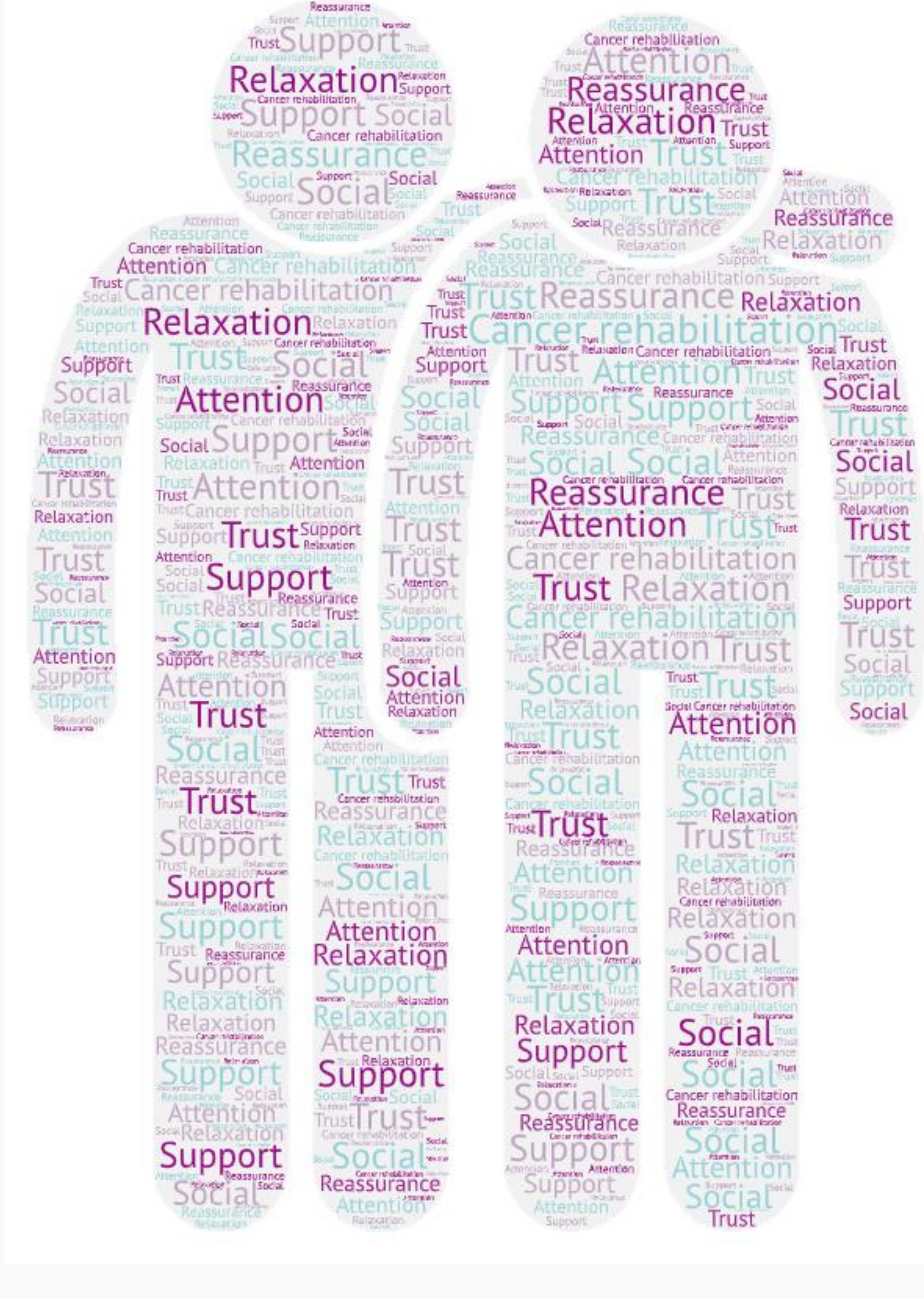

Lack of information on services as a barrier People often faced difficulties in trying to find the right services that could help with their rehabilitation needs. There was often insufficient information available for people affected by cancer to make an informed decision on who they could turn to.

'...if you speak to people they'll say 'Oh, well, I've just had aromatherapy or acupuncture or something...' And you're like 'Oh, well, I didn't know that you can get that.' 'Oh, if you need to speak to this person and this...' You know, so it's seems to be more word of mouth, there doesn't seem to be a formal kind of way of finding things out.' (Participant_37 affected by breast cancer)

'As I say, it would be nice, certainly if we had more information about [third sector] nurses, you know, because there's not a lot of hm... information out there in... in... as my opinion. Because they do such a good job, that it... you know, do they only do the job in the gym and in the ward? I don't know...

(Companion_16 partner of a person affected by prostate cancer)
Rehabilitation is not routine in the cancer pathway

Healthcare professionals reported numerous barriers to care provision. One of the main barriers is that cancer rehabilitation is not provided routinely within the cancer pathway. This could explain why people did not always have information on available services.

"Referrals into us is probably the other big difficulty, is quite ad hoc, it's only when people know about us, so it's not routine within pathways that if you've got a cancer diagnosis you get the opportunity to see a therapist." (Professional_01 - OT)

"The [Name of the surgical unit] then transfer back out to the local team, cause it might be a few weeks before they come here, they might not come here. Hm... and the teams locally are a bit more haphazard, there's no one who, some of the community teams get referrals and phone us to ask for advice, cause they don't feel competent to deal with neuro-oncology." (Professional_02 - Speech and language therapist)

\section{Conclusion:}

The preliminary findings of this study indicate that cancer rehabilitation is highly valued by people affected by cancer. However, rehabilitation is still not routinely provided within the cancer pathway. This can act as a barrier to supporting people with cancer related health problems, particularly if there is scarce knowledge on available cancer rehabilitation services. Service promotion among people affected by cancer and within the multidisciplinary team may help in overcoming this barrier.

\section{Implications:}

The qualitative study reported here is part of a three phase project: Realist Evaluation of Cancer Rehabilitation Services in South Wales (REEACaRS). Within REEACaRS qualitative and quantitative methods are combined to explore in what circumstances cancer rehabilitation services work, for whom and how. Identifying the value and barriers to cancer rehabilitation can aid the improvement of services and might help more people cope better with the consequences of cancer.

Feedback is given to the two specialist cancer rehabilitation services on the value and barriers to care provision.

Contact: Judit Csontos via e-mail: CsontosJK@Cardiff.ac.uk Date of preparation: 10/10/2019

References:

Braun, V. \& Clarke, V. (2006). Qualitative Research in Psychology, 3(2), 77-101.

Hunter et al. (2017). The American Journal of Occupational Therapy, 72(2), 1-11.

Welsh Government (2017). Welsh Cancer Patient Experience Survey. 